\title{
Preocupaciones de adolescentes de Lima y sus estilos de afrontamiento
}

\author{
Patricia Martínez Uribe \\ Roxanna Morote Ríos ${ }^{2}$ \\ Pontificia Universidad Católica del Perú
}

\begin{abstract}
Combinando metodología cualitativa y cuantitativa, el estudio describe las preocupaciones principales de un grupo de adolescentes y sus estrategias de afrontamiento comparándolas en función a variables sociodemográficas (edad, sexo y tipo de colegio). Se trata de un estudio descriptivo con un diseño transeccional en el que participaron 413 adolescentes de 13 a 18 años. El instrumento usado fue la forma específica de la Escala de Afrontamiento para Adolescentes (ACS) (Frydenberg y Lewis, 1997) que incluyó una pregunta abierta sobre su principal preocupación. Se encontró que sus mayores preocupaciones se centran en su futuro y su rendimiento escolar y que las estrategias de afrontamiento más usadas fueron Preocuparse y Esforzarse. Se encontró que las variables sociodemográficas marcan diferencias importantes entre los diferentes grupos.

Palabras clave: adolescencia, afrontamiento, preocupaciones.
\end{abstract}

\section{Concerns and coping styles in adolescents from Lima}

Combining qualitative and quantitative methodology, the study describes the main concerns of a group of adolescents and the strategies they use to cope with such concerns according to socio-demographic variables (age, sex and type of school). It is a descriptive study, with a transsectional design, of 413 adolescents from 13 to 18 years. The used instrument was the specific form of the Adolescent Coping Scale (ACS) (Frydenberg \& Lewis, 1997) that included one open question regarding their main concern. It was found that their major concerns are centered in their future and school performance and that the most used coping strategies were Concern and Work Hard. It was found that socio-demographic variables make important differences among the different groups.

Keywords: adolescence, coping, concerns.

1 Profesora asociada de la sección de Psicología del Departamento de Humanidades de la PUCP. Actualmente es coordinadora de la misma. Magíster en Psicología. Correo electrónico: pmartin@pucp.edu.pe.

2 Bachiller y jefe de práctica de la sección de Psicología de la PUCP, miembro del área de Formación del Instituto Bartolomé de las Casas. Ha realizado investigaciones en temas relacionados al desarrollo, contexto psicosocial y psicopatología en la adolescencia. Correo electrónico: roxana@bcasas.org.pe. 

El estudio de los procesos de desarrollo durante el ciclo vital ha devenido, en la actualidad, en una comprensión de la adolescencia como un periodo diferenciado y particular del desarrollo humano. En este periodo, los individuos desarrollan gran parte del repertorio de estrategias con las que enfrentarán los diversos eventos de la vida adulta (Patterson y McCubbin, 1987). La complejidad de este periodo, y las repercusiones individuales y sociales del comportamiento adolescente plantean la necesidad de estudiar los eventos que perciben como sus principales fuentes de preocupación, y los mecanismos que ponen en marcha para generar una respuesta adaptativa o disfuncional.

La visión tradicional de la adolescencia como un periodo de confusión y caos ha sido modificada progresivamente. Al considerar detenidamente la cantidad e intensidad de los cambios a los que se ven expuestos los(as) adolescentes y, más aun, cuando puede incorporarse a este conocimiento el valor subjetivo que ellos(as) confieren a sus preocupaciones y a su malestar, se puede concluir que lo que anteriormente se veía como turbulencia es un intenso proceso de adaptación, donde es necesario considerar los recursos o estrategias que desarrollan los(as) jóvenes, el costo emocional que conllevan, los posibles riesgos a los que se enfrentan, y el impacto y respuesta familiar y social que genera.

El comportamiento adolescente se encuentra inscrito dentro del marco de un conjunto de tareas de desarrollo cuyo logro supone bienestar subjetivo y salud psicológica para el individuo. En 1990, Freire (Peñaherrera, 1998) describe las siguientes tareas: la internalización de una nueva imagen corporal; el logro de la independencia económica, social y emocional de los padres; el logro de una identidad auténtica, realista y estable; la incorporación de un sistema de valores flexibles; la perspectiva del futuro que comprende aspiraciones, elección vocacional y percepción de habilidades que consoliden 
la independencia y finalmente, el desarrollo de una identidad psicosexual. Los(as) jóvenes se enfrentan a estas tareas en un periodo de tiempo relativamente corto y aunque puede considerarse que estas no se concluyen de forma definitiva, la adolescencia constituye un periodo clave en el que puede preverse la aparición de conductas de riesgo o de malestar emocional.

Finalmente, se considera que la comprensión de las demandas o fuentes de estrés de los (as) adolescentes y los mecanismos que ponen en marcha para afrontarlas debe integrar las características del medio social en el que se desarrollan. En nuestro medio existen condiciones de pobreza y violencia que se reflejan en las limitadas posibilidades de acceso a niveles superiores de estudio o a espacios laborales que logren satisfacer las demandas económicas de la familia (Panfichi, 1993). Por otra parte, los adultos no siempre reconocen o incentivan las capacidades de los jóvenes, no les brindan un soporte emocional adecuado y no reconocen su responsabilidad en las dificultades que pueden encontrar a lo largo de su desarrollo (Peñaherrera, 1998).

\section{Preocupaciones durante la adolescencia y estilos de afrontamiento}

Frydenberg (1997) señala que muchas conductas antisociales como el consumo de drogas, los trastornos de alimentación y la violencia son el resultado de la incapacidad de algunos(as) adolescentes para afrontar sus preocupaciones. El interés por identificar las preocupaciones centrales de este grupo ha llevado a algunos autores a clasificarlas dentro de dos amplios grupos: las preocupaciones globales y las preocupaciones personales. Las primeras se refieren a temas de naturaleza social (preocupaciones como el bienestar de la comunidad y del otro) mientras que las segundas corresponden a la realidad inmediata del individuo (como preocuparse por el propio bienestar emocional o material). Las llamadas preocupaciones sociales (como 
la pobreza y la violencia social) representan una visión más amplia de las dificultades del entorno y posiblemente, del rol del individuo como miembro de su comunidad.

Seiffge-Krenke (1995) realizó uno de los estudios más amplios acerca de las preocupaciones cotidianas de los(as) adolescentes sobre la base de información de más de 3000 jóvenes de países como Alemania, Israel, Finlandia y EE.UU. Encontró que los problemas relacionados con la visión del futuro son considerados los estresores de mayor intensidad: temores acerca del desempleo, inseguridad en la elección vocacional y preocupaciones generales como el medio ambiente. La autora coincide con Phelps y Jarvis (1994) al señalar que las mujeres presentan mayores preocupaciones referidas a sí mismas, sus padres o amigos y a su conducta interpersonal o conflictos con ellos. Los varones por su parte, refieren mayor tensión en temas como la escuela y actividades extracurriculares académicas y de ocio.

Existe escasa información acerca de las preocupaciones específicas de los(as) adolescentes según grupos de edad. Sin embargo, Compas et al. (Canessa, 2000) señalan fuentes de estrés diferentes según grupos etáreos: los(as) adolescentes tempranos (alrededor de 14 años) consideran que la familia es la fuente de preocupación más intensa, para los(as) adolescentes medios (entre 15 y 17 años) es el grupo de pares, mientras que para los(as) adolescentes tardíos (más de 17 años) las preocupaciones académicas son las que generan mayor malestar.

El desarrollo de diversos estilos de afrontamiento durante la adolescencia parece estar influenciado por los patrones de socialización a los que se ven expuestos las mujeres y los varones. En el caso de las mujeres, parece reproducirse la apertura social y familiar hacia la expresión de sentimientos y la comunicación, aunque también se encuentra presente el uso de la fantasía frente a los problemas. Por el contrario, los varones son alentados socialmente a la inhibición de 
sus emociones y al desarrollo de su autonomía al mismo tiempo que intensifican el uso de estrategias evitativas de afrontamiento. Así, existen evidencias del uso de estrategias distintas entre varones y mujeres. Frydenberg y Lewis (1993), Halstead, Bennett Jonson y Cunningham (1993) y Phelps y Jarvis (1994) encontraron mediante la utilización de las Escalas de Afrontamiento para Adolescentes (ACS) de Frydenberg y Lewis (1997) que las mujeres utilizan más las estrategias de búsqueda de soporte social, hacerse ilusiones, reducción de la tensión, reinterpretación de la situación, aceptación y el apoyo de la religión. Phelps y Jarvis (1994) señalan que las mujeres no solo usan más la estrategia de búsqueda de apoyo social sino que además esta estrategia tiene tanto fines instrumentales como emocionales. Los autores señalan que los varones utilizan de forma más frecuente las estrategias de realizar actividades de distracción física, la retirada, el humor y el consumo de drogas o alcohol.

A pesar de las frecuentes diferencias de género encontradas, Frydenberg y Lewis (1993) señalan que ambos grupos utilizan de forma similar estrategias de resolución directa del problema (concentrarse en resolver el problema); lo que diferencia a hombres y mujeres es la realización paralela de actividades ajenas a la situación problemática en el caso de los hombres, mientras que las mujeres esperan que suceda lo mejor de forma positiva.

\section{Metodología}

La presente investigación es de tipo descriptivo con un diseño transeccional descriptivo, ya que se obtuvo la información en un momento determinado a través de una única aplicación (Hernández, Fernández y Baptista, 1998). Sus objetivos son describir el uso de estrategias de afrontamiento, evaluadas según la Escala de Afrontamiento para Adolescentes (Frydenberg y Lewis, 1997), y las preocupaciones principales que refieren un grupo de adolescentes de Lima. Asimismo, 
se trata de comparar dichas estrategias y preocupaciones en función de variables sociodemográficas como sexo, edad y tipo de colegio.

\section{Participantes}

El grupo total del presente estudio estuvo constituido por 413 adolescentes entre los 13 y los 18 años, de la ciudad de Lima (Cuadro 1). La selección de los colegios que participaron se realizó de manera intencional. Se hizo un esfuerzo deliberado por obtener una muestra en función a criterios de sexo y edad (Kerlinger, 1988) y se eligió dos colegios particulares (203 adolescentes) y dos nacionales (210 adolescentes) de uno de los distritos con mayor cantidad de colegios de Lima Metropolitana. Al interior de los mismos, la selección de alumnos se realizó de manera incidental (Buendía, Colás y Hernández, 1998) con aquellos alumnos de $2^{\circ}$ a $5^{\circ}$ de secundaria que aceptaron participar de la investigación.

\section{Cuadro 1}

Distribución de la muestra por edad y sexo

\begin{tabular}{|lccc|}
\hline Sexo & 13 a 15 años & 16 a 18 años & Total \\
\hline Hombres & 113 & 67 & 180 \\
Mujeres & 136 & 97 & 233 \\
Total & 249 & 164 & 413 \\
\hline
\end{tabular}

\section{Instrumentos}

Se utilizó la forma específica de las Escalas de Afrontamiento para Adolescentes (ACS) de Frydenberg y Lewis (1997). El instrumento incluye una pregunta abierta, en la que se pide a los (as) adolescentes que describan brevemente la preocupación principal de sus vidas y 79 ítemes de tipo likert. Los autores reportan un coeficiente de confiabilidad test-retest (producto momento de Pearson) superior a 
0,32 y validez de constructo sobre la base del análisis factorial que apoya la existencia de las 18 estrategias de afrontamiento. Casullo y Fernández (2000) reportan en una muestra de 1320 adolescentes argentinos que la prueba obtiene una consistencia interna de .85 (alfa) y confiabilidad de .7 y .8 con el método de división por mitades.

En el Perú, Canessa (2000) reporta para la forma general del ACS un nivel de confiabilidad ítem-test mayor a .20 para 78 de los 79 ítemes. Señala también que el análisis de consistencia interna revela valores alfa comprendidos entre .48 y .84 lo que indica que todas las escalas del ACS son confiables. En cuanto a la validez de constructo, Canessa replicó el procedimiento de análisis de Frydenberg y Lewis (1997), confirmando los hallazgos de los autores. Las - 18 escalas que constituyen la prueba pueden ser agrupadas en tres categorías generales que se presentan en el Cuadro 2.

\section{Cuadro 2}

Escalas del ACS

\begin{tabular}{|ll|}
\hline Categorías generales & \multicolumn{1}{|c|}{ Escalas } \\
\hline Afrontamiento positivo & Fijarse en lo positivo (Po) \\
& Concentrarse en resolver el problema (Rp) \\
& Buscar diversiones Relajantes (Dr) \\
& Distracción física (Fi) \\
& Esforzarse y tener éxito (Es) \\
\hline Búsqueda de otros & Buscar apoyo Social (As) \\
& Invertir en amigos íntimos (Ai) \\
& Buscar Pertenencia (Pe) \\
& Buscar apoyo espiritual (Ae) \\
& Buscar ayuda profesional (Ap) \\
& Acción social (So) \\
\hline Estrategias menos efectivas & Preocuparse (Pr) \\
& Hacerse ilusiones (Hi) \\
& Falta de afrontamiento (Na) \\
& Reducción de la tensión (Rt) \\
& Ignorar el problema (lp) \\
& Autoinculparse (Cu) \\
& Reservarlo para sí (Re) \\
\hline
\end{tabular}


Preocupaciones de adolescentes de Lima y sus estilos de afrontamiento

\section{Procedimiento}

La aplicación se realizó en las aulas de $2^{\circ}$ a $5^{\circ}$ de secundaria de los colegios seleccionados, de manera colectiva con dos examinadores en cada una de ellas. Luego se procedió al análisis de datos en dos etapas: en primer término se realizó el análisis de contenido de la pregunta abierta acerca de la preocupación principal de los(as) adolescentes, la unidad de registro escogida fue el tema ya que es una unidad de significación compleja, de longitud variable y su realidad es de origen psicológico (Bardin, 1992). Esto permitió identificar núcleos de sentido agrupados en las siguientes categorías: personal, futuro, escuela, pares, familia, social y diversión (Cuadro 3).

\section{Cuadro 3}

Categorías de análisis de la Preocupación Principal

\begin{tabular}{|c|c|}
\hline Categoría & Contenidos \\
\hline Personal & $\begin{array}{l}\text { - Sentimientos de soledad, abandono, pérdida o tristeza } \\
\text { - Miedos y temores } \\
\text { - Dificultades con la autoimagen o autoestima }\end{array}$ \\
\hline Futuro & $\begin{array}{l}\text { - Laboral. Ser profesional, tener éxito y dinero } \\
\text { - Vocacional. Incertidumbre sobre la carrera } \\
\text { - Familiar. Cuidado de la familia }\end{array}$ \\
\hline Escuela & - Calificaciones, temor a reprobar cursos \\
\hline Pares & $\begin{array}{l}\text { - Amigos, problemas específicos, preocupación por ellos } \\
\text { - Pareja, dificultades, deseo de pareja }\end{array}$ \\
\hline Familia & $\begin{array}{l}\text { - Problemas económicos al interior de la familia } \\
\text { - Problemas de relación, comunicación o comprensión } \\
\text { - Violencia, desintegración familiar, abandono }\end{array}$ \\
\hline Social & - El futuro del país o del mundo. Política. Pobreza \\
\hline Diversión & - Vacaciones, formas de diversión \\
\hline
\end{tabular}

\section{Resultados}

En primer lugar, se presentarán las respuestas de los(as) adolescentes a la pregunta de aquello que constituye su preocupación principal 
actual, luego se presentarán los puntajes obtenidos en las escalas de afrontamiento. En ambos casos se muestran las diferencias significativas encontradas en función al sexo, edad y nivel socioeconómico.

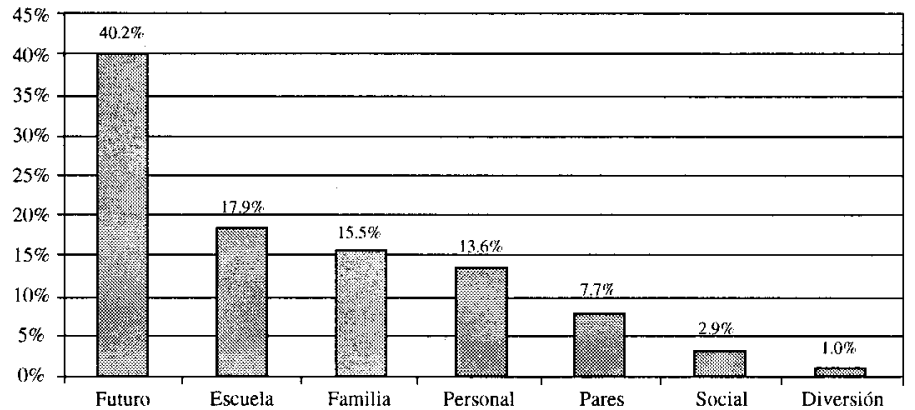

Figura 1. Preocupación principal en el grupo total.

En la Figura 1 se observan las frecuencias y porcentajes de aquello que constituye la principal preocupación para el grupo en general.

Las preocupaciones ante el futuro son mencionadas por el $40.2 \%$ del grupo total, al interior de esta categoría se encuentra que la preocupación principal se refiere al futuro laboral o vocacional, que incluye al $\mathbf{9 2 . 8 \%}$ de estos casos:

- Algo que me preocupa es qué va a ser de mí en el futuro. Aún no decido qué carrera estudiar, me gustan varias y no sé decidirme. A veces me siento mal al saber que otras personas de mi edad ya están decididas acerca de su futuro (mujer, 15 años, colegio nacional).

- Tener trabajo y vivir como gente (hombre, 16 años, colegio particular).

- Me preocupa qué voy a estudiar en la universidad, quiero que después de estudiar tenga un buen empleo y con un buen salario para así mantenerme a mí y a mi familia (mujer, 13 años, colegio particular). 
- La principal es cómo estaré en mis estudios, trabajos, si me irá bien en todo, cómo será, porque yo quiero triunfar, pero lamentablemente la crisis de estos últimos tiempos, la pobreza que se agranda deja en neutro muchos proyectos que tengo para lograr mis objetivos, pero sé que con esfuerzo y dedicación lo voy a lograr (mujer, 16 años, colegio particular).

- Terminar la secundaria, ingresar a una universidad y al término de esto no conseguir trabajo, no por falta de capacidad sino por el desempleo (hombre, 16 años, colegio nacional).

En segundo lugar se encuentran las preocupaciones por el rendimiento escolar (17.9\%), asociadas en general a las calificaciones en la escuela o a dificultades con algún o algunos cursos en particular, por ejemplo:

- Que siempre he tenido muchos rojos y mi papá siempre me ha pegado, pero nunca he repetido de año; yo estudio mucho pero se me olvida (hombre, 13 años, colegio nacional).

- La preocupación que yo tengo es cuando salgo mal en mis notas $y$ me es difícil superarlas (hombre, 16 años, colegio nacional).

Luego, aparecen las preocupaciones por la familia, que se presentan en $15.5 \%$ del grupo total. El $50 \%$ de estas respuestas se refieren a dificultades de comunicación al interior de la familia y el otro $50 \%$ a preocupaciones económicas, desorganización o desintegración familiar:

- Lidiar con mis papás para que me dejen salir con mis amigos, siento que ellos son como mi familia y puedo confiar en ellos como en nadie antes. El que mis papás los detesten solo hace que yo los deteste a ellos (mujer, 14 años, colegio particular).

- El gran problema de mi vida es no tener una casa, un hogar donde vivir pero eso va a desaparecer porque yo estudiaré y trabajaré para conseguirlo y vivir junto a mi mamá (mujer, 15 años, colegio nacional). 
- Las broncas con mi mamá; ella y yo somos muy diferentes, casi nunca estamos de acuerdo (mujer, 15 años, colegio nacional).

- Las discusiones de mis padres; me preocupa que estos afecten a mis hermanos; y la larga espera de ver, creo, cuando llegará el día de irme (mujer, 14 años, colegio nacional).

- La situación económica de la familia, pues genera constantes conflictos (mujer, 14 años, colegio particular).

Las preocupaciones personales (13.6\%) son aquellas referidas fundamentalmente a sí mismos. Aquí encontramos que el $26.8 \%$ se refieren a sentimientos de soledad y desamparo, el $46.4 \%$ a miedos y temores de diversa índole y el $26.8 \%$ son preocupaciones referidas a su autoimagen:

- Salir adelante en la vida, mi gran preocupación es no ser nadie en la vida (hombre, 17 años, colegio particular).

- Quedarme solo, sin amigos, sin trabajo y que mi familia me rechace o no me quiera. Quedarme solo sin nadie que me ayude o quiera (hombre, 14 años, colegio particular).

- Siento que no soy nadie y que no debería existir (hombre, 14 años, colegio particular).

- Yo quiero ser otra persona para no tener que ser humillada por otras personas (mujer, 17 años, colegio nacional).

- Perder a los que más quiero, que se alejen porque no estén bien conmigo, que no les caiga o que no me soporten, ya sea en la casa, colegio, con los amigos, y estar sola y triste; no me gusta que me den bajones (mujer, 14 años, colegio nacional).

- Que a veces soy muy cruel con las personas (hombre, 18 años, colegio nacional).

En la categoría de amigos y pareja $(7.7 \%)$, predominan las preocupaciones por los amigos con un $84.4 \%$ del total de este grupo. Las respuestas en este caso se refieren esencialmente a preocupaciones por lograr más o mejores amistades o a peleas y desilusiones con amigos o amigas. 
Preocupaciones de adolescentes de Lima y sus estilos de afrontamiento

- Hace un tiempo que no me hablo con una compañera por el motivo que a las dos nos gusta el mismo chico. Ella tiene enamorado y a mí ya no me interesa el chico, pero todavía hay discordia entre las dos. Nos hablamos de vez en cuando, pero ya no hay la misma confianza como antes (mujer, 14 años, colegio nacional).

- Lo que me importa más por ahora son los amigos. Primero les caigo bien, pero tal vez más tarde ya no; ¿que me pasaría? no sé, pero quiero mejorar mi vida (hombre, 14 años, colegio nacional).

- En este momento lo que más me "preocupa" es el hecho de salir del colegio y enfrentarme con otro mundo diferente. Sobre todo, el hecho de dejar de ver a muchas personas que son mis amigos (hombre, 16 años, colegio particular).

Un área que encontramos particularmente importante es la de preocupaciones de tipo social, vinculadas al país o al mundo. Si bien la cantidad de jóvenes que la describe es solo un $2.9 \%$ de casos, su presencia resulta significativa. Por ejemplo:

- Lo que más me preocupa en este país es la pobreza en que vivimos, que la gente pobre es siempre marginada y las personas que tienen plata creen que son lo máximo, cuando realmente no son nada ya que son soberbios. También me preocupan los niños de la calle o abuelitos que no tienen a nadie; también los perritos que no tienen qué comer y los tratan como basura (mujer, 16 años, colegio nacional).

- Creo que me preocupa el mundo en general; el trabajo, porque hoy en día vemos carencia de valores y todo aquello que vemos en la televisión, la falta de trabajo, la economía del país está mal, cada día para mí hay más gente que no tiene principios. A mí me preocupa esto porque mi futuro está en este país tan mal (mujer, 15 años, colegio nacional).

- El problema que más me afecta es el mundo de hoy. Me pongo a pensar y digo por qué tanta muerte, violencia, injusticia, hambre, miseria. Porque si nosotros queremos podemos cambiar. Vivimos un calvario (mujer, 13 años, colegio nacional). 
- El mundo en general, creo yo porque hay tanta gente que es enferma y que buscan hacer daño, o simplemente hay gente suelta dañina que es muy peligrosa y tú no puedes vivir en paz y no estás libre de nada (mujer, 16 años, colegio particular).

Finalmente en la categoría de diversión, en solo un 1\% del grupo, se encuentran preocupaciones con relación a cómo pasar las vacaciones o el fin de semana, o a diferentes formas de diversión, como computadora o juegos de vídeo.

Al comparar las preocupaciones de los jóvenes de acuerdo con las variables de sexo, edad y tipo de colegio, se encuentran diferencias estadísticamente significativas a través de la prueba de Chi cuadrado, en las tres variables.

\section{Cuadro 4}

Preocupación principal de acuerdo con el sexo, edad y tipo de colegio

\begin{tabular}{|l|cc|cc|cc|}
\hline & \multicolumn{2}{|c|}{$\begin{array}{c}\text { Sexo* } \\
\%\end{array}$} & \multicolumn{2}{c|}{ Edad** } & \multicolumn{2}{c|}{ Colegio*** } \\
& Hombres & Mujeres & $13-15$ & $16-18$ & Particular & Nacional \\
\hline Futuro & 36.1 & $\mathbf{4 3 . 3}$ & 34.5 & $\mathbf{4 8 . 8}$ & $\mathbf{4 4 . 3}$ & 36.2 \\
Escuela & $\mathbf{2 3 . 3}$ & 13.7 & $\mathbf{2 0 . 1}$ & 14.6 & 16.7 & $\mathbf{1 9}$ \\
Familia & $\mathbf{1 7 . 2}$ & 14.2 & $\mathbf{1 8 . 5}$ & 11 & 11.8 & $\mathbf{1 9}$ \\
Personal & 13.3 & 13.7 & 11.6 & $\mathbf{1 6 . 5}$ & $\mathbf{1 5 . 8}$ & 11.4 \\
Pares & 4.4 & $\mathbf{1 0 . 3}$ & $\mathbf{9 . 6}$ & 4.9 & - & - \\
Social & 1.7 & $\mathbf{3 . 9}$ & - & - & 1 & $\mathbf{4 . 8}$ \\
Diversión & - & - & - & - & 2 & - \\
\hline
\end{tabular}

${ }^{*} X^{2}(7, N=413)=19.89 p=.005 ; * * X^{2}(7, N=413)=15.99 p=.025$;

$* * * X^{2}(7, N=413)=21.15 p=.003$

Las mujeres (Cuadro 4) presentan mayores preocupaciones en relación con su futuro, a sus amistades (pares) y a lo que sucede en el país (social). Loś hombres destacan en mayor preocupación por su rendimiento escolar (escuela) y por la familia, en particular por la economía familiar. Al interior de la categoría familia, las mujeres destacan en cuanto a la preocupación por las relaciones familiares y 
la desorganización familiar. Asimismo, al interior de la categoría de preocupaciones personales, las mujeres presentan mayores temores que los hombres.

Con respecto a la edad, los(as) adolescentes menores, de 13 a 15 años presentan mayor preocupación por su rendimiento escolar, por la familia y por los amigos. Asimismo, al interior de la categoría de preocupaciones personales, el grupo de 13 a 15 años presenta una preocupación significativamente mayor por sentimientos de soledad y abandono. Los(as) mayores, de 16 a 18 años, muestran mayor preocupación por su futuro y por cuestiones personales.

Con relación al tipo de colegio, que puede ser considerado como un indicador del nivel socioeconómico, puede observarse que los(as) alumnos de colegio particular destacan en presentar mayores preocupaciones por el futuro, por sí mismos (personal) y por la diversión. En los de colegio nacional predomina la preocupación por el rendimiento escolar (escuela), por la familia y por el país (social).

\section{Cuadro 5}

Estrategias de afrontamiento del grupo total

\begin{tabular}{|lcc|}
\hline \multicolumn{1}{|c}{ Escalas } & $M$ & $D E$ \\
\hline Buscar apoyo social (As) & 64.38 & 15.61 \\
Concentrarse en resolver el problema (Rp) & 69.73 & 13.74 \\
Esforzarse y tener éxito (Es) & 71.67 & 13.52 \\
Preocuparse (Pr) & 77.66 & 12.08 \\
Invertir en amigos íntimos (Ai) & 65.15 & 15.04 \\
Buscar pertenencia (Pe) & 67.57 & 12.05 \\
Hacerse ilusiones (Hi) & 58.77 & 13.89 \\
Falta de afrontamiento (Na) & 41.69 & 13.22 \\
Reducción de la tensión (Rt) & 35.52 & 12.54 \\
Acción social (So) & 39.07 & 13.31 \\
Ignorar el problema (Ip) & 39.59 & 14.78 \\
Autoinculparse (Cu) & 49.58 & 15.54 \\
Reservarlo para sí (Re) & 51.32 & 15.61 \\
Buscar apoyo espiritual (Ae) & 56.24 & 13.51 \\
Fijarse en lo positivo (Po) & 67.84 & 13.68 \\
Buscar ayuda profesional (Ap) & 52.70 & 18.47 \\
Buscar diversiones relajantes (Dr) & 69.90 & 15.48 \\
Distracción física (Fi) & 66.57 & 18.88 \\
\hline
\end{tabular}


Los puntajes obtenidos en las escalas de afrontamiento del ACS en el grupo total se presentan en el Cuadro 5. Aquí se observa que la estrategia más usada por el grupo en general es de las menos efectivas: Preocuparse, seguida por Esforzarse y tener éxito, y Concentrarse en resolver el problema, que son las más efectivas.

En la comparación de grupos a partir de las variables sexo, edad y tipo de colegio (Cuadro 6) se observan diferencias significativas $(p<.05)$ en varias escalas a través de la Prueba de Análisis de Varianza (ANOVA).

Las escalas Reservarlo para sí (Re) y Concentrarse en resolver el problema ( $\mathrm{Rp}$ ) presentan diferencias significativas en los tres grupos de comparación. Acción social (So), Ignorar el problema (Ip), Reservarlo para sí $(\mathrm{Re})$ y Distracción física (Fi) muestran puntajes más altos en los hombres mientras que las escalas de Buscar apoyo social (As) y Concentrarse en resolver el problema (Rp) muestran puntajes más elevados en el caso de las mujeres.

Las diferencias de acuerdo con la edad señalan puntajes superiores en el grupo de mayor edad (16 a 18 años). Los alumnos de colegios particulares obtienen puntajes superiores en las escalas de Buscar apoyo social (As), Concentrarse en resolver el problema (Rp), Invertir en amigos íntimos (Ai), Buscar Pertenencia $(\mathrm{Pe})$ y Buscar diversiones Relajantes (Dr); mientras que los de colegios nacionales, en las escalas de Reservarlo para sí (Re) y Buscar apoyo espiritual (Ae). 
Preocupaciones de adolescentes de Lima y sus estilos de afrontamiento

\section{Cuadro 6}

Estrategias de afrontamiento de acuerdo con el sexo, edad y tipo de colegio*

\begin{tabular}{|c|cc|cc|cc|}
\hline \multirow{2}{*}{ Escalas } & \multicolumn{2}{|c|}{ Sexo } & \multicolumn{2}{c|}{ Edad } & \multicolumn{2}{c|}{ Colegio } \\
\cline { 2 - 7 } & Hombres & Mujeres & $13-15$ & $16-18$ & Particular & Nacional \\
\hline $\mathrm{As}$ & 61.23 & 66.80 & & & 68.10 & 60.76 \\
$\mathrm{Rp}$ & 67.04 & 71.79 & 67.97 & 72.42 & 71.37 & 68.13 \\
$\mathrm{So}$ & 41.23 & 37.40 & & & & \\
$\mathrm{Ip}$ & 42.82 & 37.10 & & & & \\
$\mathrm{Re}$ & 53.35 & 49.76 & 49.72 & 53.77 & 48.94 & 53.64 \\
$\mathrm{Fi}$ & 71.33 & 62.91 & & & & \\
$\mathrm{Es}$ & & & 70.52 & 73.42 & & \\
$\mathrm{Pr}$ & & & 76.64 & 79.21 & & \\
$\mathrm{Ai}$ & & & 63.85 & 67.13 & 69.05 & 61.38 \\
$\mathrm{Rt}$ & & & 33.71 & 38.28 & & \\
$\mathrm{Cu}$ & & & 47.93 & 52.08 & & \\
$\mathrm{Pe}$ & & & & & 70.03 & 65.17 \\
$\mathrm{Ae}$ & & & & & 73.22 & 59.16 \\
$\mathrm{Dr}$ & & & & & & \\
\hline
\end{tabular}

${ }^{*} p<.05$

\section{Discusión}

Al iniciar el análisis de las respuestas dadas por los(as) jóvenes acerca de sus preocupaciones principales, encontramos la necesidad de elaborar un análisis de contenido específico, diferente al utilizado en un estudio similar realizado en Argentina (Casullo y Fernández, 1999). A pesar de hallar que en el caso de las estrategias de afrontamiento, los resultados reportados son, en líneas generales, comparables a los argentinos, las particularidades del grupo de adolescentes de Lima quedaron establecidas desde el inicio.

Los(as) adolescentes de Lima, de forma similar a lo encontrado por Seiffge-Krenke (1995), señalan como su preocupación principal problemas relacionados al futuro. La alta proporción de estas respuestas nos remite a lo que podemos considerar como un mito en nuestro medio: que el comportamiento de riesgo en adolescentes proviene de una orientación en el presente sumada a la falta de reflexión 
acerca de su porvenir. En los(as) adolescentes, sobre todo en las mujeres, la preocupación por acceder a niveles superiores de estudio (sobre todo universitarios) es muy alta y atribuyen a ellos la facultad de otorgarles un estatus social altamente valorado. Sorprende la frecuente analogía entre ser profesional y ser alguien y la descripción de esta idea como triunfo, reconocimiento social, solvencia económica y con ello, no defraudar a sus padres. De esto se desprende que los(as) adolescentes reflejan en sus respuestas la influencia de estereotipos y valores familiares que se traducen en fuentes de demanda intrínseca. Encuentran que su valoración dentro del núcleo familiar va a ser producto de la posibilidad de contribuir al sustento económico de la familia y, en muchos casos, al cuidado de padres y hermanos.

Por otro lado, a pesar de que existe la idea de éxito asociada a la profesión, aparece de forma muy clara la preocupación acerca de poder conseguir un trabajo que consolide dicho éxito. Al parecer, los(as) adolescentes se encuentran muy alertas a las condiciones sociales que los rodean, no pierden de vista que las condiciones de desempleo son, en la actualidad, una de las características más generalizadas en nuestra sociedad y que alcanza a grupos económicos de mayor y menor ingreso. Estas condiciones facilitan que el logro profesional sea una fuente de estrés intenso en los(as) adolescentes (sobre todo en los mayores). Resulta preocupante reconocer que las condiciones de nuestra sociedad no puedan responder a las expectativas de los(as) adolescentes ya que, como señalan Marceli, Braconnier y Ajuriaguerra (1986), el fracaso en la búsqueda de empleo es vivido como un fracaso de la propia imagen social (independientemente de que existan esas condiciones sociales desfavorables) y puede generar un desequilibrio afectivo cuyas consecuencias son sentimientos de fracaso, de rechazo de la sociedad, impresión de inutilidad del tiempo en el colegio y riesgo de marginalidad.

La segunda fuente de estrés mencionada con mayor frecuencia se refiere a su éxito académico actual, sobre todo los varones de menor 
edad (entre 13 y 15 años) de colegios nacionales. Las descripciones de los adolescentes refieren en muchos casos sentimientos de inadecuación o incapacidad para afrontar estas dificultades. El fracaso escolar es considerado un factor de riesgo en el desarrollo de los(as) adolescentes. Peñaherrera (1998) señala que favorece conductas de riesgo como la deserción escolar, la violencia y el consumo de drogas. Los(as) adolescentes lo describen como un aspecto que disminuye su estima personal y al parecer, como un problema que no encuentra en la escuela fuentes de soporte.

Entre los(as) adolescentes que refieren los conflictos de relación y económicos dentro del núcleo familiar como su principal preocupación encontramos que los(as) de menor nivel socioeconómico y edad lo hacen con mayor frecuencia. Al inicio de la adolescencia existe una mayor sensibilidad a centrarse en los conflictos entre los padres lo que puede cuestionar su función como fuente de soporte y protección. Seiffge-Krenke (1995) señala que la percepción de un clima familiar desfavorable facilita la aparición de conductas de afrontamiento más disfuncionales. Del mismo modo, es posible que a esta edad se incremente la necesidad de autonomía y exista una mayor búsqueda de los pares como fuente de soporte. En relación con este proceso, se encuentra que la dificultad de los padres para afrontar esta demanda de autonomía aparece en las descripciones de los(as) adolescentes como una fuente importante de conflicto. Por otra parte, el tipo de preocupaciones familiares que se encuentran con mayor frecuencia en hombres y en mujeres es diferente: la necesidad de una mayor comunicación y de un medio más integrado es más intensa en las mujeres; para los hombres, la preocupación principal es la carencia económica de la familia. La socialización diferenciada asociada al género se reproduce en esta área, ya que mientras para la mujer la preservación de los vínculos afectivos es central, la tensión asociada a las necesidades prácticas y la posible asimilación de responsabilidades económicas es más frecuente en los varones. 
A diferencia de lo encontrado en la mayoría de estudios revisados sobre el tema, observamos un considerable porcentaje de jóvenes cuya preocupación central se refiere a conflictos internos, generalmente de tipo emocional y que aparecen descritos como una fuente de malestar de gran intensidad. En primer término, analizamos una serie de respuestas en las que la percepción de soledad, abandono o carencia afectiva se presenta como una realidad inmediata o como una posibilidad futura. Los sentimientos asociados al deterioro real o anticipado de vínculos nos indica la posible presencia de síntomas depresivos en estos(as) jóvenes. Seiffge-Krenke (1995) reporta que los(as) adolescentes con una estructura de personalidad depresiva así como los que muestran síntomas depresivos son más propensos a presentar estrategias de afrontamiento disfuncionales; además, señala que los síntomas depresivos aparecen asociados a otros síndromes clínicos como ansiedad, trastornos de conducta y de alimentación, así como a la percepción de un medio familiar adverso en los(as) adolescentes.

La categoría de preocupaciones referidas a relaciones con pares (amigos y parejas) muestra que los(as) jóvenes no encuentran en las relaciones de pareja una fuente de estrés considerable. Los conflictos con pares son descritos en términos de temor a perder amigos(as) y a la desilusión en relación con la imagen que tenían de las personas con las que han tenido dificultades. Aquí los resultados son muy similares a los descritos en otros países ya que son las mujeres las que claramente encontrarían en sus relaciones interpersonales un factor de soporte importante por lo que los conflictos en esta área les genera una tensión mayor a la registrada por los varones.

En cuanto a las preocupaciones de carácter social, encontramos temas referidos a la pobreza, el desempleo y grupos marginales de la sociedad. En este caso, es notoria la alta frecuencia de este tipo de respuestas en mujeres provenientes de niveles socioeconómicos menos favorecidos. Si bien la literatura sobre el tema señala que el compromiso con el entorno social es un factor subyacente a este tipo de 
respuestas, una lectura detenida de los contenidos encontrados muestra que podrían existir mecanismos de identificación en este grupo con las personas menos favorecidas de la sociedad. Es posible que las mujeres que viven en condiciones económicas adversas perciban que tienen menos posibilidades de acceder a puestos de trabajo o que la familia no podrá impulsar sus estudios superiores lo que las coloca en una posición de mayor vulnerabilidad. Su crítica a la sociedad no deja de tener componentes de realidad, pero se encontraría influenciada por sus condiciones personales de vida.

El análisis de los resultados obtenidos sobre la utilización de estrategias de afrontamiento señala que existen muchos puntos de coincidencia con las muestras española (Frydenberg y Lewis, 1997), argentina (Buenos Aires) (Casullo y Fernández, 1999) y de un grupo de Lima (Canessa, 2000), así como algunos puntos de diferencia. En todos los casos dentro del grupo de estrategias menos utilizadas se encuentran Reducción de la tensión (Rt), Ignorar el problema (Ip) y Acción social (So); las dos primeras son consideradas por Frydenberg y Rowley (1998) como estrategias menos efectivas y dirigidas al manejo emocional. En nuestro caso, la Falta de Afrontamiento (Na) también es una estrategia poco usada lo que señalaría que los(as) adolescentes estudiados tienden a evitar el uso de estrategias de afrontamiento consideradas disfuncionales. Consideramos que el limitado uso de la estrategia de Acción social puede relacionarse a que los(as) adolescentes no percibirían redes de soporte social efectivas, con las que podrían organizar una acción conjunta dirigida a resolver sus preocupaciones.

Las estrategias más utilizadas en nuestro caso y en el grupo español son Esforzarse y tener éxito (Es) y Preocuparse (Pr), mientras la primera corresponde al grupo de estrategias efectivas y dirigidas al problema, la segunda se encuentra entre las estrategias dirigidas al manejo emocional. Estos datos nos señalan que los(as) jóvenes responden simultáneamente a la demanda y al estrés que esta genera. Es 
decir, la acción práctica no se desliga de manifestaciones de temor y ansiedad, como lo describe la escala Pr. Otra característica de esta escala es su orientación hacia el futuro tema que como vimos es la fuente de estrés más importante en el grupo estudiado.

La escala Buscar apoyo espiritual (Ae), se presenta de manera similar a lo encontrado por Canessa (2000). Al parecer, los jóvenes en Lima, sobre todo los que provienen de medios socioeconómicos de mayor carencia, encuentran en la religión una fuente de ayuda que sería característica de nuestro contexto. Además de considerar factores culturales, podría tomarse en cuenta que en nuestro medio existe una amplia red de agrupaciones religiosas cuyo trabajo se orienta, en muchos casos, a brindar ayuda material a las personas.

En cuanto a las diferencias de género podemos señalar que el grupo estudiado muestra diferencias significativas solo en seis escalas de la prueba a diferencia del grupo estudiado en Buenos Aires donde doce escalas presentan puntajes estadísticamente diferentes. Al parecer, en nuestro grupo, el comportamiento de hombres y mujeres tiene mayores semejanzas. Analizando los resultados presentados por Canessa (2000), Casullo y Fernández (1999) y los expuestos en la presente investigación, vemos que las diferencias de género se confirman en las escalas de Ignorar el problema (Ip) y Distracción física (Fi) en favor de los hombres y de Buscar apoyo social (As) en favor de las mujeres. Las dos escalas descritas con mayor frecuencia en varones suponen conductas que no actúan directamente sobre la situación estresante, sin embargo, la distracción física es vista por Frydenberg y Rowley (1998) como una forma de afrontamiento positivo ya que se refiere al desarrollo de actividades saludables.

En el caso de las mujeres, se confirma el uso de habilidades de comunicación. Es posible inferir que una percepción femenina del medio, como un espacio donde se puede encontrar fuentes de apoyo y bienestar o donde puede establecerse un intercambio emocional, 
facilita el uso de este tipo de estrategias de afrontamiento. La sensibilidad descrita por las mujeres en relación con los problemas de comunicación dentro de la familia, podría relacionarse a este resultado (la percepción de este tipo de problemas familiares podría restarle una importante fuente de apoyo a las mujeres). También encontramos, a diferencia de otros estudios, que los varones usan con mayor frecuencia la estrategia de Reservarlo para sí (Re), es decir, tienden a aislarse y a no dar a conocer sus preocupaciones. Es decir, en esta área, hombres y mujeres presentan marcadas diferencias en sus comportamientos.

Sumado a esto, encontramos que, a diferencia de los resultados reportados por Frydenberg y Lewis (1993), los estudios en contextos latinoamericanos señalan que las mujeres usan de forma más frecuente la escala Concentrarse en resolver el problema (Re). Esta es una de las escalas cuyo tipo de afrontamiento de la situación resulta más positivo ya que se dirige al análisis de la situación y al desarrollo sistemático de actividades para solucionarla. Podría así suponerse que las mujeres podrían estar presentando múltiples enfoques frente a una situación problemática y que la búsqueda del otro como fuente de ayuda no supone necesariamente una actitud pasiva frente a una demanda.

La comparación de los grupos de edad muestra que los(as) adolescentes de mayor edad usan con mayor frecuencia formas más variadas de afrontamiento ya que las estrategias que reportan un mayor uso pertenecen a los tres grupos generales: afrontamiento positivo (Rp, Es), búsqueda de soporte social (Ai) y manejo emocional ( $\mathrm{Pr}, \mathrm{Rt}, \mathrm{Cu}, \mathrm{Re}$ ). Sin embargo, cabe destacar que hay un uso mayor de estrategias del tercer grupo. Es posible que los(as) jóvenes de mayor edad hayan alcanzado niveles mayores de madurez, lo que les permite un manejo más directo de sus emociones frente a situaciones problemáticas.

En cuanto a diferencias atribuidas al nivel socioeconómico de los (as) jóvenes, vemos que los(as) adolescentes que estudian en colegios particulares realizan un afrontamiento más efectivo $(R p)$ y muestran 
una orientación mayor a establecer relaciones interpersonales como fuente de soporte (As, Ai, Pe). En líneas generales, las diferencias favorecen a este grupo ya que los(as) jóvenes de niveles socioeconómicos menos favorecidos tienden al aislamiento (Re) y al afrontamiento religioso (Ae). Es posible asociar estos resultados a las condiciones del medio social; podemos mencionar condiciones como la pobreza, la desigualdad de oportunidades y la calidad de los servicios educativos como factores que intervienen de forma negativa en el desarrollo de estos(as) adolescentes (Peñaherrera, 1998).

Los hallazgos de este estudio señalan la importancia de tomar en cuenta aquello que los(as) jóvenes consideran como su preocupación principal, con el fin de desarrollar, a partir de ello, programas de intervención que contemplen tanto el área educacional como el área clínica: la primera dirigida a una orientación vocacional que considere las condiciones reales del medio social y cultural en el que se desarrollan los(as) jóvenes y la segunda, dirigida al manejo de los estresores familiares y emocionales de los(as) adolescentes. Ambas áreas aparecen como potenciales factores de riesgo en el desarrollo de la juventud en nuestro medio. La intervención dirigida a potencializar el desarrollo de estrategias de afrontamiento debe considerar al grupo de hombres y a los que se encuentran en condiciones socioeconómicas menos favorecidas, como poblaciones que requieren desarrollar estrategias más adaptativas frente a las demandas del medio.

\section{Referencias}

Bardin, D. (1992). El análisis de contenido. Buenos Aires: Mc GrawHill.

Buendía, L., Colás, P. y Hernández, F. (1998). Métodos de Investigación en Psicopedagogía. Madrid: Mc Graw-Hill.

Canessa, B. (2000). Adaptación de la prueba "Escalas de afrontamiento para adolescentes" en escolares entre 14 y 17 años de 
edad de diferente sexo y nivel socioeconómico. Tesis para optar el título de Licenciada en Psicología no publicada, Universidad de Lima, Lima.

Casullo, M. y Fernández, M. (2000). Estrategias de Afrontamiento en estudiantes adolescentes (Monografía de investigación). Buenos Aires: Universidad de Buenos Aires.

Frydenberg, E. y Lewis, R. (1993). Boys play sports and girls turn to others: age, gender, and ethnicity as determinants of coping. Journal of Adolescence, 16, 253 - 266.

Frydenberg, E. (1997). Adolescent coping: Theoretical and research perspectives. Londres: Routledge

Frydenberg, E. y Lewis, R. (1997). Manual: Escalas de afrontamiento para adolescentes (ACS). Madrid: TEA.

Frydenberg, E. y Rowley, G. (1998). Coping with social issues: What Australian university students do. Issues in Educational Research, 8 (1), 33-48.

Halstead, M., Bennett Johnson, S. y Cunningham, W. (1993). Measuring coping in adolescents: An application of the ways of coping checklist. Journal of Clinical Child Psychology, 22 ( 3), 337-344.

Hernández, R., Fernández, C. y Baptista, P. (1998). Metodología de la investigación. México: Mc Graw-Hill.

Kerlinger, F. (1988). Investigación del comportamiento. México DF: Mc Graw-Hill.

Marcelli, D., Braconnier, A. y Ajuriaguerra, J. (1986). Manual de psicopatología del adolescente. Barcelona: Masson.

Panfichi, A. (1993). Juventud, tradición y trabajo. En G. Portocarrero (Ed.). Los nuevos limeños (pp. 285-312). Lima: Tafos Sur.

Patterson, J. y McCubbin, H. (1987). Adolescent coping style and behaviours: Conceptualization and measurement. Journal of Adolescence, 10, 163-86.

Peñaherrera, E. (1998). Comportamiento de riesgo adolescente: una aproximación psicosocial. Revista de Psicología de la PUCP, $2,16,265-293$. 
Phelps, S. y Jarvis, P. (1994). Coping in adolescence: Empirical evidence for a theoretically based approach to assessing coping. Journal of Youth and Adolescence, 23 (3), 359 - 371.

Seiffge-Krenke, I. (1995). Stress, coping and relationships in adolescence. Nueva Jersey: Erlbaum. 